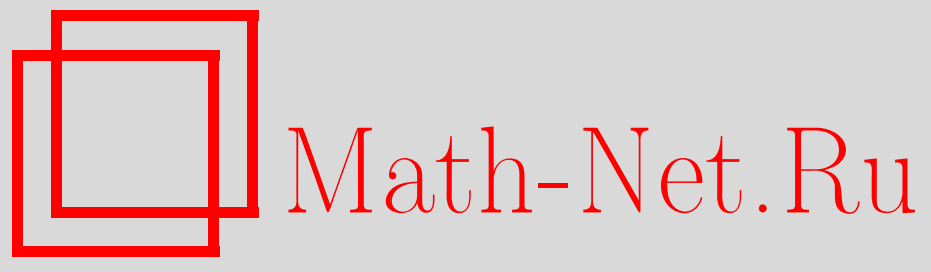

В. В. Катрахов, Ю. Н. Харченко, Двумерные четырехлинейные модели типа модели Изинга, ТМФ, 2006, том 149, номер 2, 281-298

DOI: https://doi.org/10.4213/tmf4234

Использование Общероссийского математического портала Math-Net.Ru подразумевает, что вы прочитали и согласны с пользовательским соглашением http://www . mathnet.ru/rus/agreement

Параметры загрузки:

IP: 54.224 .187 .69

26 апреля 2023 г., 09:43:18

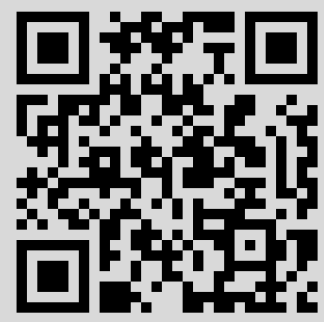




\title{
ДВУМЕРНЫЕ ЧЕТЫРЕХЛИНЕЙНЫЕ МОДЕЛИ ТИПА МОДЕЛИ ИЗИНГА
}

\begin{abstract}
Кратко изложена теория корневых трансфер-матриц для четырехлинейных моделей с полем, причем в новой безындексной форме. Теоретическими и численными методами обнаружены новые эффекты в теории сингулярных точек и фазовых переходов. Значительная часть результатов получена с помощью численного алгоритма, основанного на чрезвычайной разреженности корневой трансфер-матрицы, который уменьшает вычислительную сложность моделей типа модели Изинга радикальным (по крайней мере экспоненциальным) образом.
\end{abstract}

Ключевые слова: модель Изинга, трансфер-матрица, статистическая сумма, ферромагнетик, антиферромагнетик, свободная энергия, намагниченность, магнитная восприимчивость, критические точки, сингулярные кривые, фазовые переходы.

\section{1. СТАТИСТИЧЕСКАЯ СУММА, СВОБОДНАЯ ЭНЕРГИЯ И КОРНЕВАЯ ТРАНСФЕР-МАТРИЦА}

С современным состоянием общей теории решеточных моделей статистической механики можно ознакомиться в [1]. В книге [2] введено понятие корневой трансферматрицы (KTM).

\section{1. Схема четырехлинейного взаимодействия и статистическая сумма.} Рассмотрим на плоскости квадратную решетку с узлами в точках с натуральными координатами $(n, m)$, где $n=1, \ldots, N+1, m=1, \ldots, M+1, N, M$ - некоторые натуральные числа. В каждом узле рассматривается бинарная переменная (спин) $\sigma_{n}^{m}$, которая принимает значения \pm 1 . Каждый спин взаимодействует с восемью ближайшими спинами по четырем направлениям или линиям (отсюда и название "четырехлинейные" магнетики): по горизонтали, вертикали и двум диагоналям. Такая схема взаимодействия соответствует наложению друг на друга двух решеток, одна из которых (прямая) образована серией вертикальных и горизонтальных линий, другая (диагональная) образована двумя сериями диагональных линий.

* Институт прикладной математики ДВО РАН, Владивосток, Россия. E-mail: katrakhov@mail.ru 
В двумерной четырехлинейной модели на решетке рассматривается статистическая сумма, имеющая вид

$$
\begin{aligned}
Z_{N M}^{(4)}\left(K_{1}, K_{2}, K_{3}, K_{4}, H\right)=\sum_{\sigma} & \prod_{n=1}^{N} \prod_{m=1}^{M} \exp \left(K_{1} \sigma_{n}^{m} \sigma_{n+1}^{m}+K_{2} \sigma_{n}^{m} \sigma_{n}^{m+1}+\right. \\
& \left.+K_{3} \sigma_{n+1}^{m} \sigma_{n}^{m+1}+K_{4} \sigma_{n}^{m} \sigma_{n+1}^{m+1}+H \sigma_{n}^{m}\right) .
\end{aligned}
$$

Здесь суммирование производится по всем состояниям спинов, параметры $K_{i}, i=$ $1,2,3,4$, суть параметры межспинового взаимодействия по $i$-й линии взаимодействия. Они имеют вид $K_{i}=k_{i} /(B T)$, где $k_{i}$ - коэффициент межспинового взаимодействия по той же линии, $B$ - постоянная Больцмана, $T$ - температура; $H=$ $h /(B T)$ - параметр взаимодействия с внешним полем с коэффициентом $h$.

Заметим, что $K_{1}, K_{2}$ являются параметрами взаимодействия по горизонтали и вертикали (мы их будем называть первой группой параметров), а $K_{3}, K_{4}$ - параметрами взаимодействия по двум диагоналям (вторая группа параметров).

Отметим, что для корректности определения статистической суммы следует задать условия на граничные спины. Однако никакие краевые условия на граничные спины не влияют на величину свободной энергии, которая определяется ниже (см., например, [2], [3]). Опираясь на это, введем и всюду в дальнейшем будем считать выполненными следующие краевые условия:

$$
\sigma_{n}^{M+1}=\sigma_{n}^{1}, \quad n=1,2, \ldots, N ; \quad \sigma_{N+1}^{m}=\sigma_{1}^{m+1}, \quad m=1,2, \ldots, M .
$$

Таким образом, мы ввели тороидальные краевые условия по вертикальному направлению решетки и условия, сдвинутые на единицу, по горизонтальному направлению.

Свободная энергия $F^{(4)}$, которую здесь мы представим в некотором нормированном виде, определяется как термодинамический предел:

$$
F^{(4)}=F^{(4)}\left(K_{1}, K_{2}, K_{3}, K_{4}, H\right)=\lim _{N, M \rightarrow \infty} \frac{\ln Z_{N M}^{(4)}}{N M} .
$$

Приравнивая нулю межспиновое взаимодействие по некоторым направлениям (линиям), мы получаем магнетики меньших линейностей: так, полагая $K_{4}=0$, что соответствует трехлинейному магнетику или модели типа модели Изинга на треугольной решетке, мы получаем его свободную энергию $F^{(3)}\left(K_{1}, K_{2}, K_{3}, H\right)=$ $F^{(4)}\left(K_{1}, K_{2}, K_{3}, 0, H\right)$. Соответственно в случае двухлинейного магнетика свободная энергия $F^{(2)}\left(K_{1}, K_{2}, H\right)=F^{(4)}\left(K_{1}, K_{2}, 0,0, H\right)$. В книге [2] доказано, что можно приравнять нулю не только последние параметры межспинового взаимодействия, но и любые из них, например,

$$
\begin{aligned}
F^{(3)}\left(K_{1}, K_{2}, K_{3}, H\right) & =F^{(4)}\left(K_{1}, K_{2}, K_{3}, 0, H\right)=F^{(4)}\left(K_{1}, K_{2}, 0, K_{3}, H\right)= \\
& =F^{(4)}\left(K_{1}, 0, K_{2}, K_{3}, H\right)=F^{(4)}\left(0, K_{1}, K_{2}, K_{3}, H\right) .
\end{aligned}
$$

Применительно к соответствующим параметрам корневых трансфер-матриц этот факт имеет нетривиальный характер. 
1.2. Корневая трансфер-матрица четырехлинейной модели. С помощью известной процедуры [1], [2] вводится трансфер-матрица (ТМ) размера $2^{N} \times 2^{N}$. Свободная энергия выражается через главное собственное значение (ГСЗ) $\Lambda_{\mathrm{TM}}$ по формуле

$$
F^{(4)}=\lim _{N \rightarrow \infty} \frac{\ln \Lambda_{\mathrm{TM}}}{N}
$$

(главным называется положительное однократное и максимальное по модулю собственное значение).

Оказывается, что из ТМ можно извлечь в явном виде корень степени $N$, эту матрицу в дальнейшем мы называем корневой ТМ, или сокращенно КТМ.

Для записи КTM нам потребуются четыре однопараметрические матрицы $U=$ $U(X), V=V(X), W=W(X), G=G(X)$ размера $8 \times 8$, имеющие, соответственно, вид

$$
\begin{aligned}
& U=\operatorname{diag}\left(e^{X}, e^{-X}, e^{-X}, e^{X}, e^{X}, e^{-X}, e^{-X}, e^{X}\right) \\
& V=\operatorname{diag}\left(\left(\begin{array}{cc}
e^{X} & e^{-X} \\
e^{-X} & e^{X}
\end{array}\right),\left(\begin{array}{cc}
e^{X} & e^{-X} \\
e^{-X} & e^{X}
\end{array}\right),\left(\begin{array}{cc}
e^{X} & e^{-X} \\
e^{-X} & e^{X}
\end{array}\right),\left(\begin{array}{cc}
e^{X} & e^{-X} \\
e^{-X} & e^{X}
\end{array}\right)\right), \\
& W=\operatorname{diag}\left(e^{X}, e^{-X}, e^{X}, e^{-X}, e^{-X}, e^{X}, e^{-X}, e^{X}\right) \\
& G=\operatorname{diag}\left(e^{X}, e^{-X}, e^{X}, e^{-X}, e^{X}, e^{-X}, e^{X}, e^{-X}\right)
\end{aligned}
$$

Для любой матрицы $Q$ размера $8 \times 8$ через $Q_{\mathrm{b}}$ будем обозначать блочно-диагональную матрицу размера $2^{N} \times 2^{N}$, блоками которой служат матрицы $Q$.

В дальнейшем постоянно будет использоваться матрица $\boldsymbol{P}_{N}$ размера $2^{N} \times 2^{N}$, являющаяся матрицей в каноническом базисе $\delta^{j}=\left(\delta_{1}^{j}, \ldots, \delta_{2^{N}}^{j}\right)^{\mathrm{T}}$ (здесь $\delta_{i}^{j}-$ символ Кронекера, Т - знак транспонирования) известного оператора перестановок (пермутатора), переставляющего векторы канонического базиса в следующем порядке: $\delta^{j} \rightarrow \delta^{2 j-1}, \delta^{2^{N-1}+j} \rightarrow \delta^{2 j}, j=1, \ldots, 2^{N-1}$.

ПРЕДЛОЖЕНИЕ 1.

1. $N$-я степенъ матрици $R^{(4)}=Q_{\mathrm{b}}^{(4)} \boldsymbol{P}_{N}$, где $Q^{(4)}=U\left(K_{1}\right) V\left(K_{3}\right) U\left(K_{2}\right) W\left(K_{4}\right) \times$ $G(H)$, является TM четырехлинейной модели, т.е. матрица $R^{(4)}$ является КТМ этой модели.

2. Существует ГСЗ $\Lambda_{R}^{(4)}$ KTM $R^{(4)}$ такое, что свободная энергия вычисляется по формуле

$$
F^{(4)}=\lim _{N \rightarrow \infty} \ln \Lambda_{R^{(4)}}
$$

ДоКАЗАТЕЛЬСТВо этого базового утверждения опирается на общую теорию KTM, изложенную с помощью специально разработанной техники индексирования матриц в книге [2]. Отсюда, учитывая заданные в неявной форме краевые условия, мы можем извлечь вид КТМ. В работе [4] эта неявная форма краевых условий трансформирована в краевые условия (2). Поскольку эти рассуждения громоздки и занимают много места, за деталями мы отсылаем читателя к указанным работам. 


\section{2. ЭЛЕМЕНТЫ ТЕОРИИ КТМ ЧЕТЫРЕХЛИНЕЙНОЙ МОДЕЛИ}

В этом разделе мы рассмотрим некоторые элементы теории КTM, которые потребуются для классификации типов магнетиков. Эта классификация приведет, в частности, к значительному сокращению объема вычислительной работы.

2.1. Общие положения. Для моделей с полем справедливы следующие утверждения.

ПрЕДЛОЖЕНИЕ 2. Все четыре КТМ, полученные независимыми перестановками параметров $K_{1} \leftrightarrow K_{2}$ и $K_{3} \leftrightarrow K_{4}$, эквивалентны в термодинамическом пределе:

$$
\begin{aligned}
F^{(4)}\left(K_{1}, K_{2}, K_{3}, K_{4}, H\right) & =F^{(4)}\left(K_{2}, K_{1}, K_{3}, K_{4}, H\right)= \\
& =F^{(4)}\left(K_{1}, K_{2}, K_{4}, K_{3}, H\right)=F^{(4)}\left(K_{2}, K_{1}, K_{4}, K_{3}, H\right) .
\end{aligned}
$$

ДоКАЗАТЕЛЬСтво предложения с помощью методов теории КТМ является чрезвычайно громоздким, вместе с тем это утверждение почти очевидно вследствие симметрии (в термодинамическом пределе, который к тому же не зависит от вида краевых условий) статистической суммы относительно поворотов на угол $90^{\circ}$ и отражения относительно прямых и диагональных линий решетки.

ПРЕДЛОЖЕНИЕ 3. Изменение в КТМ знака параметра H приводит к подобной матриче.

Утверждения подобного рода доказывались в работах [5] для моделей меньших линейностей, схема доказательства полностью подходит и для четырехлинейных магнетиков. Оно идентично доказательству того, что статсумма и свободная энергия являются четными функциями параметра взаимодействия с полем.

Для моделей без поля справедливо

ПрЕДЛОЖЕНИЕ 4. Для абсолютно изотропных магнетиков, т.е. для случая $\left|K_{i}\right|=K, i=1,2,3,4$, имеет место серия соотношений вида

$$
\begin{aligned}
F^{(4)}(K, K, K, K, 0) & =F^{(4)}(-K, K,-K,-K, 0)= \\
& =F^{(4)}(K,-K,-K,-K, 0)=F^{(4)}(-K,-K, K, K, 0), \\
F^{(4)}(-K,-K,-K,-K, 0) & =F^{(4)}(K,-K, K, K, 0)= \\
& =F^{(4)}(-K, K, K, K, 0)=F^{(4)}(K, K,-K,-K, 0), \\
F^{(4)}(K, K, K,-K, 0) & =F^{(4)}(K,-K, K,-K, 0)= \\
& =F^{(4)}(-K, K, K,-K, 0)=F^{(4)}(-K,-K, K,-K, 0)= \\
& =F^{(4)}(K, K,-K, K, 0)=F^{(4)}(K,-K,-K, K, 0)= \\
& =F^{(4)}(-K, K,-K, K, 0)=F^{(4)}(-K,-K,-K, K, 0) .
\end{aligned}
$$

ДокАЗАтЕльство. Для доказательства мы вынуждены обратится к индексированной форме представления КТМ, поскольку изложение в безындексной форме весьма затруднительно. Напомним, что с развитой авторами техникой индексирования можно ознакомиться в книге [2]. 
Простейший пермутатор имеет вид

$$
\boldsymbol{P}_{2}=P=\left(\begin{array}{llll}
1 & 0 & 0 & 0 \\
0 & 0 & 1 & 0 \\
0 & 1 & 0 & 0 \\
0 & 0 & 0 & 1
\end{array}\right)
$$

при этом

$$
\boldsymbol{P}_{N}=P_{[N 1]} P_{[(N-1) 1]} \cdot \ldots \cdot P_{[21]} \cdot
$$

Введем две матрицы с параметром и одну из матриц Паули:

$$
\begin{gathered}
A=A(X)=\operatorname{diag}\left(e^{X}, e^{-X}, e^{-X}, e^{X}\right) \\
B=B(X)=\left(\begin{array}{cc}
e^{X} & e^{-X} \\
e^{-X} & e^{X}
\end{array}\right), \quad Y=\left(\begin{array}{ll}
0 & 1 \\
1 & 0
\end{array}\right) .
\end{gathered}
$$

Тогда КТМ в индексированной форме выглядит следующим образом:

$$
R^{(4)}=A_{[21]}\left(K_{1}\right) B_{[1]}\left(K_{3}\right) A_{[21]}\left(K_{2}\right) A_{[31]}\left(K_{4}\right) \boldsymbol{P}_{N} .
$$

Имеют место следующие коммутационные соотношения:

$$
\begin{aligned}
Y_{[1]} A_{[21]}(X) & =A_{[21]}(-X) Y_{[1]}, & Y_{[2]} A_{[21]}(X) & =A_{[21]}(-X) Y_{[2]}, \\
Y_{[1]} A_{[31]}(X) & =A_{[31]}(-X) Y_{[1]}, & Y_{[3]} A_{[31]}(X) & =A_{[31]}(-X) Y_{[3]}, \\
Y_{[1]} B_{[1]}(X) & =B_{[1]}(X) Y_{[1]}, & Y_{[i]} \boldsymbol{P}_{N} & =\boldsymbol{P}_{N} Y_{[i-1]},
\end{aligned}
$$

кроме того,

$$
Y_{[1]} B_{[1]}(X)=B_{[1]}(-X) .
$$

Напомним, что матрицы с несовпадающими индексами коммутируют. Из этих соотношений вытекает важное коммутационное соотношение

$$
Y_{[1]} Y_{[2]} \ldots Y_{[N]} R^{(4)}=R^{(4)} Y_{[1]} Y_{[2]} \ldots Y_{[N]}
$$

Рассмотрим сначала случай четного $N$. В этом случае справедливы соотношения

$$
\begin{aligned}
& Y_{[1]} Y_{[3]} Y_{[5]} \ldots Y_{[N-1]} R^{(4)} Y_{[1]} Y_{[3]} Y_{[5]} \ldots Y_{[N-1]}= \\
& \quad=Y_{[1]} Y_{[3]} Y_{[5]} \ldots Y_{[N-1]} A_{[21]}\left(K_{1}\right) B_{[1]}\left(K_{3}\right) A_{[21]}\left(K_{2}\right) A_{[31]}\left(K_{4}\right) Y_{[2]} Y_{[4]} Y_{[6]} \ldots Y_{[N]} \boldsymbol{P}_{N}= \\
& \quad=Y_{[1]} Y_{[2]} Y_{[3]} \ldots Y_{[N]} A_{[21]}\left(-K_{1}\right) B_{[1]}\left(K_{3}\right) A_{[21]}\left(-K_{2}\right) A_{[31]}\left(K_{4}\right) \boldsymbol{P}_{N} .
\end{aligned}
$$

С одной стороны, матрица в правой части последнего равенства подобна $\mathrm{KTM} R^{(4)}$, а с другой стороны, вследствие коммутационного соотношения (7) ее ГСЗ совпадает с ГС3 матрицы $R^{(4)}$, что в силу связи ГСЗ и свободной энергии означает выполнение (предельного) соотношения

$$
F^{(4)}\left(K_{1}, K_{2}, K_{3}, K_{4}, 0\right)=F^{(4)}\left(-K_{1},-K_{2}, K_{3}, K_{4}, 0\right) .
$$


Рассмотрим теперь случай нечетного $N$. В этом случае справедливы соотношения

$$
\begin{aligned}
& Y_{[1]} Y_{[3]} Y_{[5]} \ldots Y_{[N]} R^{(4)} Y_{[1]} Y_{[3]} Y_{[5]} \ldots Y_{[N]}= \\
& \quad=Y_{[1]} Y_{[3]} Y_{[5]} \ldots Y_{[N]} A_{[21]}\left(K_{1}\right) B_{[1]}\left(K_{3}\right) A_{[21]}\left(K_{2}\right) A_{[31]}\left(K_{4}\right) Y_{[2]} Y_{[4]} Y_{[6]} \ldots Y_{[N-1]} Y_{[1]} \boldsymbol{P}_{N}= \\
& =Y_{[1]} Y_{[2]} Y_{[3]} \ldots Y_{[N]} A_{[21]}\left(-K_{1}\right) B_{[1]}\left(K_{3}\right) Y_{[1]} A_{[21]}\left(K_{2}\right) A_{[31]}\left(-K_{4}\right) \boldsymbol{P}_{N}= \\
& \quad=Y_{[1]} Y_{[2]} Y_{[3]} \ldots Y_{[N]} A_{[21]}\left(-K_{1}\right) B_{[1]}\left(-K_{3}\right) A_{[21]}\left(K_{2}\right) A_{[31]}\left(-K_{4}\right) \boldsymbol{P}_{N},
\end{aligned}
$$

что означает выполнение (предельного) равенства

$$
F^{(4)}\left(K_{1}, K_{2}, K_{3}, K_{4}, 0\right)=F^{(4)}\left(-K_{1}, K_{2},-K_{3},-K_{4}, 0\right) .
$$

Из (8), (9) и (3) следует справедливость предложения.

Соответствующие утверждения для магнетиков меньших линейностей имеются в [5], [6].

2.2. Классификация магнетиков с полем. Классификацию магнетиков мы проведем только для абсолютно изотропного случая, т.е. при условии, что все параметры межспинового взаимодействия совпадают по абсолютной величине, поскольку все вычисления сделаны только для этого случая.

Отметим, что абсолютно анизотропный случай изучается по точно такой же схеме, различия между соответствующими магнетиками носят не качественный, а количественный характер.

2.2.1. Четырехлинейные магнетики. Четырехлинейные магнетики разделяются, вообще говоря, на $2^{4}=16$ классов в соответствии с упорядоченной сигнатурой параметров межспинового взаимодействия. Каждый класс соответствует аббревиатуpe $X_{1} X_{2} \| X_{3} X_{4}$, где вместо каждого $X_{i}$ независимо друг от друга мы ставим буквы $\mathrm{F}$ (ферромагнитное взаимодействие, для которого параметр $K_{i}$ положителен) или А (антиферромагнитное взаимодействие, для которого параметр $K_{i}$ отрицателен). Две вертикальные черты в аббревиатурах разделяют две группы параметров, в первой группе параметры относятся к горизонтальной и вертикальной линиям взаимодействия, во второй - к двум диагональным линиям. Такое разделение параметров на две группы необходимо из-за отсутствия симметрии между ними. Это приводит к большему количеству типов четырехлинейных магнетиков по сравнению с магнетиками меньших линейностей, у которых все параметры межспинового взаимодействия входят в свободную энергию симметричным образом.

Из предложения 2 следует, что свободная энергия и, следовательно, все остальные макрохарактеристики магнетиков в термодинамическом пределе совпадают для определенных классов магнетиков. При этом, имея в виду совпадение свободных энергий, мы можем записать следующие равенства для классов магнетиков: $\mathrm{FF}\|\mathrm{FA}=\mathrm{FF}\| \mathrm{AF}, \mathrm{FA}\|\mathrm{FF}=\mathrm{AF}\| \mathrm{FF}, \mathrm{FA}\|\mathrm{FA}=\mathrm{FA}\| \mathrm{AF}=\mathrm{AF}\|\mathrm{FA}=\mathrm{AF}\| \mathrm{AF}, \mathrm{FA} \| \mathrm{AA}=$ $\mathrm{AF}\|\mathrm{AA}, \mathrm{AA}\| \mathrm{FA}=\mathrm{AA} \| \mathrm{AF}$. В результате существуют всего девять различных (т.е. имеющих различные свободные энергии) типов магнетиков. Один тип состоит только из тех сигнатурно упорядоченных магнетиков, в которых F- и А-взаимодействия 
переставляются между собой только внутри указанных выше двух групп. Мы будем использовать полужирный шрифт для обозначения типов магнетиков, чтобы отличать их от классов магнетиков. Перечислим девять типов магнетиков: FF || $\mathbf{F F}$, FF $\|$ FA, FF $\|$ AA, FA $\|$ FF, FA $\|$ FA, FA $\|$ AA, AA $\|$ FF, AA $\|$ FA, AA $\|$ AA.

2.2.2. Трехлинейные магнетики. Трехлинейные магнетики разделяются на $2^{3}=$ 8 классов в соответствии с упорядоченной сигнатурой параметров межспинового взаимодействия.

Параметры $K_{1}, K_{2}, K_{3}$ входят в свободную энергию симметричным образом. Это можно обнаружить, во-первых, путем анализа статсуммы с помощью эквивалентного преобразования квадратной решетки в правильную треугольную решетку, во-вторых, методами теории КТМ: сравнением ГСЗ КТМ, построенных для различных порядков суммирования в статистической сумме [2]. Таким образом, не меняя свободной энергии, мы можем переставлять их между собой, следовательно, $F F A=F A F=A F F, F A A=A F A=A A F$. В результате мы имеем всего четыре различных (т.е. с различными свободными энергиями) типа трехлинейных магнетиков: FFF, FFA, FAA, AAA.

2.2.3. Двухлинейные магнетики. Двухлинейные магнетики разделяются на $2^{2}=$ 4 класса в соответствии с упорядоченной сигнатурой параметров межспинового взаимодействия. Так как параметры $K_{1}, K_{2}$ входят в свободную энергию симметричным образом, т.е. их можно переставлять между собой, и при этом свободная энергия не меняется, то мы имеем $F A=A F$. В результате существуют всего три различных типа двухлинейных магнетиков: FF, FA, AA.

2.3. Классификация магнетиков без поля. Для четырехлинейных магнетиков по предложению 4 в абсолютно изотропном случае количество различных типов магнетиков уменьшается с девяти до трех (называемых далее блоками) в соответствии с соотношениями (4)-(6). Первый блок включает магнетики типов $\mathbf{F F}|| \mathbf{F F}$, FA $\|\mathbf{A A}, \mathbf{A A}\| \mathbf{F F}$; второй - магнетики типов AA $\|\mathbf{A A}, \mathbf{F A}\| \mathbf{F F}, \mathbf{F F} \| \mathbf{A A}$; третий магнетики типов FF $\|\mathbf{F A}, \mathbf{F A}\| \mathbf{F A}, \mathbf{A A} \| \mathbf{F A}$.

Из результатов работ [5] следует, что для трехлинейных магнетиков имеется всего два блока, в первый входят магнетики типов FFF, FAA, а во второй - магнетики типов FFA, AAA; для двухлинейных магнетиков имеется всего один блок, в который входят магнетики всех возможных типов.

Таким образом справедливо

ПреДЛОЖЕНИЕ 5. В плоских L-линейных моделях без поля, $L=2,3,4$, в абсолютно изотропном случае количество блоков магнетиков равно $L-1$.

\section{3. ВЫЧИСЛИТЕЛЬНЫЕ ЭКСПЕРИМЕНТЫ}

Все численные результаты получены тем же алгоритмом, что и в работе [6], где он подробно описан. Отметим при этом, что намагниченность (частная производная 
$\partial F^{(4)} / \partial H$ свободной энергии по параметру взаимодействия с внешним полем) вычислялась нами по схеме теории возмущений, остальные производные $\left(\partial^{2} F^{(4)} / \partial H^{2}-\right.$ магнитная восприимчивость, $\partial^{2} F^{(4)} / \partial K^{2}$ - псевдотеплоемкость и др.) вычислялись по простейшим и по возможности симметричным разностным схемам.

Отдельно поясним преимущество работы с КТМ по сравнению с работой с ТМ. Для нахождения ГСЗ мы используем степенной (итерационный) метод, основной цикл которого состоит из умножения матрицы на вектор размерности $2^{N}$. Для разреженной $\mathrm{KTM} \mathrm{для} \mathrm{этого} \mathrm{требуется} 3 \cdot 2^{N}$ арифметических операций, для полностью заполненной ТМ $-2 \cdot 4^{N}$ операций. Поскольку ТМ является $N$-й степенью соответствующей KTM, то одно указанное умножение для ТМ соответствует $N$ умножениям для КТМ, следовательно, применение КТМ в экспоненциальное число раз, равное $2 \cdot 4^{N} /\left(3 N \cdot 2^{N}\right) \approx 0.7 \cdot 2^{N} / N$, эффективнее применения ТМ.

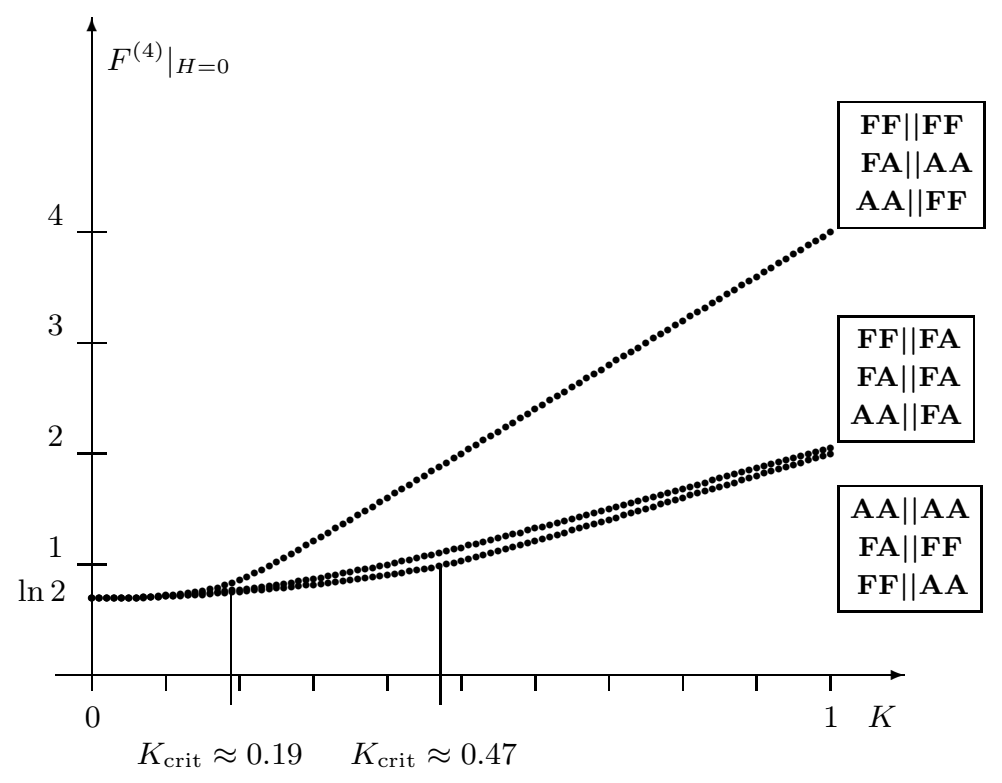

Рис. 1. Свободная энергия $F^{(4)}$ четырехлинейных магнетиков в моделях без поля.

Очень важно также то, что при использовании ТМ необходимо иметь большую оперативную память для хранения элементов полностью заполненных ТМ, то есть $4^{N}$ слов. Уже для $N$ немногим более десяти это превышает объем оперативной памяти современных персональных компьютеров. С другой стороны, КTM вообще не надо хранить в оперативной памяти. Конечно, элементы ТМ можно не хранить в памяти, а вычислять их при каждом обращении к ним, однако это приводит к недопустимо большому (в $N \cdot 4^{N}$ раз) увеличению количества арифметических операций в каждом цикле. 


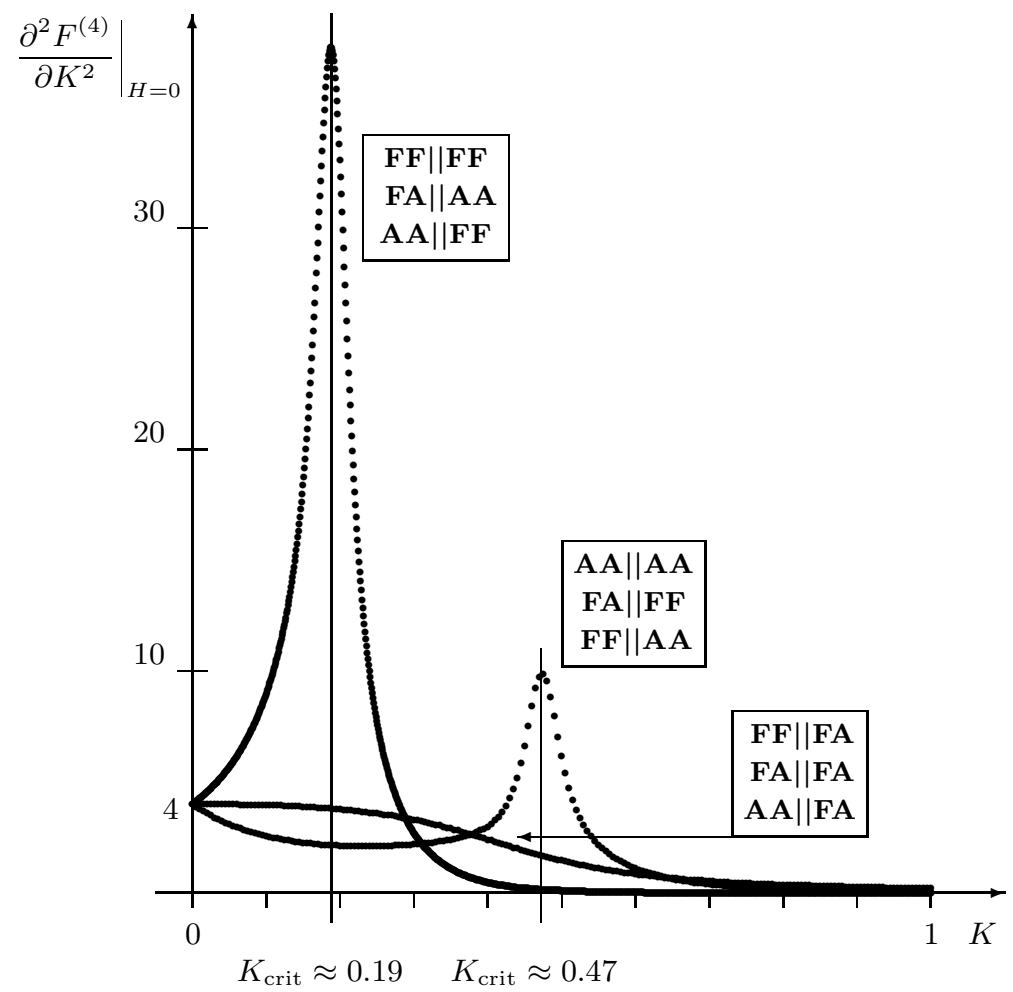

Рис. 2. Вторые производные свободной энергии $F^{(4)}$ по параметру межспинового взаимодействия $K$ для магнетиков трех блоков.

3.1. Четырехлинейные магнетики без поля. Далее приведены результаты численных экспериментов.

3.1.1. Свободная энергия. На рис. 1 мы приводим графики свободной энергии для всех трех блоков изучаемых нами магнетиков. Здесь и всюду в дальнейшем все графики построены по результатам работы нашего численного алгоритма.

3.1.2. Псевдотеплоемкость. По приведенным выше графикам свободной энергии трудно судить о ее особенностях. Поэтому для выявления критических точек мы будем исходить из того, что вторая производная от свободной энергии по параметру межспинового взаимодействия (псевдотеплоемкость) имеет сингулярность в критических точках, которая должна хорошо проявляться уже при конечных $N$.

Отметим сразу же обнаруженную точную формулу

$$
\left.\frac{\partial^{2} F^{(4)}( \pm K, \pm K, \pm K, \pm K, 0)}{\partial K^{2}}\right|_{K=0}=4
$$

аналогичная формула $\left.\frac{\partial^{2} F^{(L)}}{\partial K^{2}}\right|_{K=0}=L$ ранее была обнаружена нами и для меньших линейностей $L=0,1,2,3$.

6 Теоретическая и математическая физика, т. 149, № 2, 2006 г. 


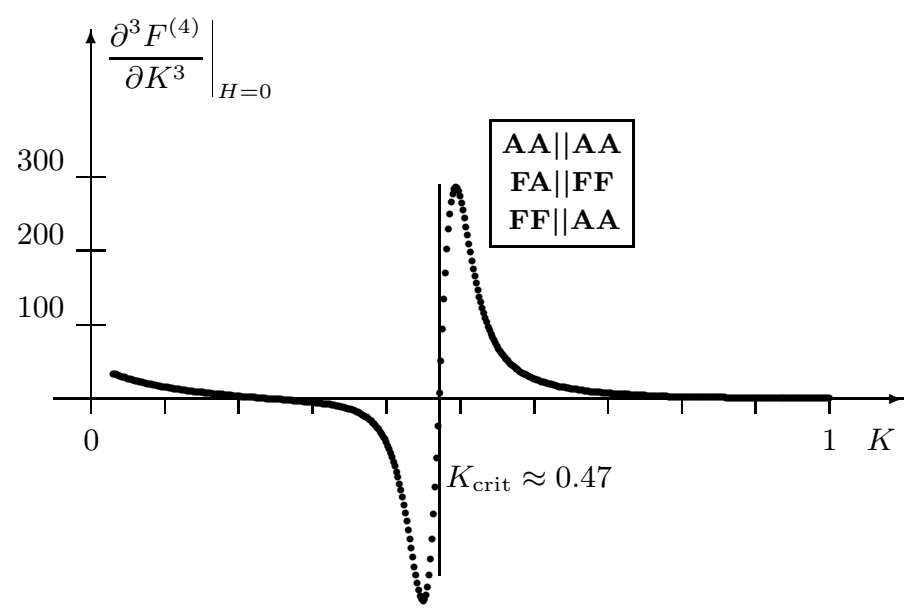

Рис. 3. Третья производная свободной энергии $F^{(4)}$ по параметру межспинового взаимодействия $K$ указанных типов магнетиков второго блока.

На рис. 2 видно, что у первого блока магнетиков имеется критическая точка $K_{\text {crit }} \approx 0.19$, у второго блока магнетиков имеется критическая точка $K_{\text {crit }} \approx 0.47$, а у третьего блока магнетиков критическая точка отсутствует.

Кроме того, в термодинамическом пределе высота пика на графике первого блока магнетиков бесконечна. На графике второго блока мы можем утверждать с гораздо меньшей уверенностью, что высота пика бесконечна, но следующая производная в критической точке имеет разрыв второго рода, о чем достаточно достоверно свидетельствует рис. 3.

Что касается магнетиков третьего блока, то все дополнительные исследования с привлечением производных вплоть до 6-го порядка показали отсутствие у них критической точки при нулевом поле.

Таким образом, мы показали, что наличие или отсутствие критической точки в моделях без поля полностью определяется сигнатурой второй группы параметров межспинового взаимодействия (относящейся к диагональным линиям взаимодействия): при сигнатурной изотропии второй группы параметров критическая точка существует, а при сигнатурной анизотропии - нет.

3.2. Намагниченность четырехлинейных магнетиков с полем. Приведем графики намагниченностей (производных $\partial F^{(4)} / \partial H$ ) всех девяти типов магнетиков, выбирая в ряде случаев различные диапазоны и шаги изменения параметров. Мы рассматриваем следующие случаи:

1) магнетик с четырьмя F-взаимодействиями (рис. 4);

2) магнетики с тремя F-взаимодействиями (рис. 5 и 6);

3) магнетики с двумя F-взаимодействиями (рис. 7-9);

4) магнетики с одним F-взаимодействием (рис. 10 и 11);

5) магнетик без F-взаимодействия (рис. 12). 


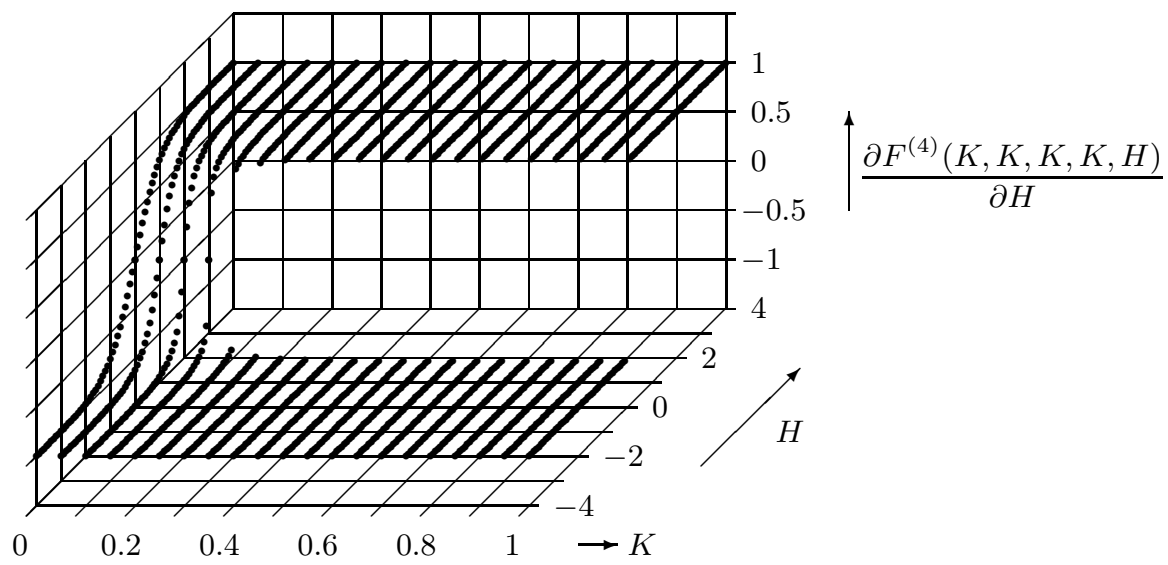

Рис. 4. Намагниченность магнетиков типа $\mathbf{F F} \| \mathbf{F F}$.

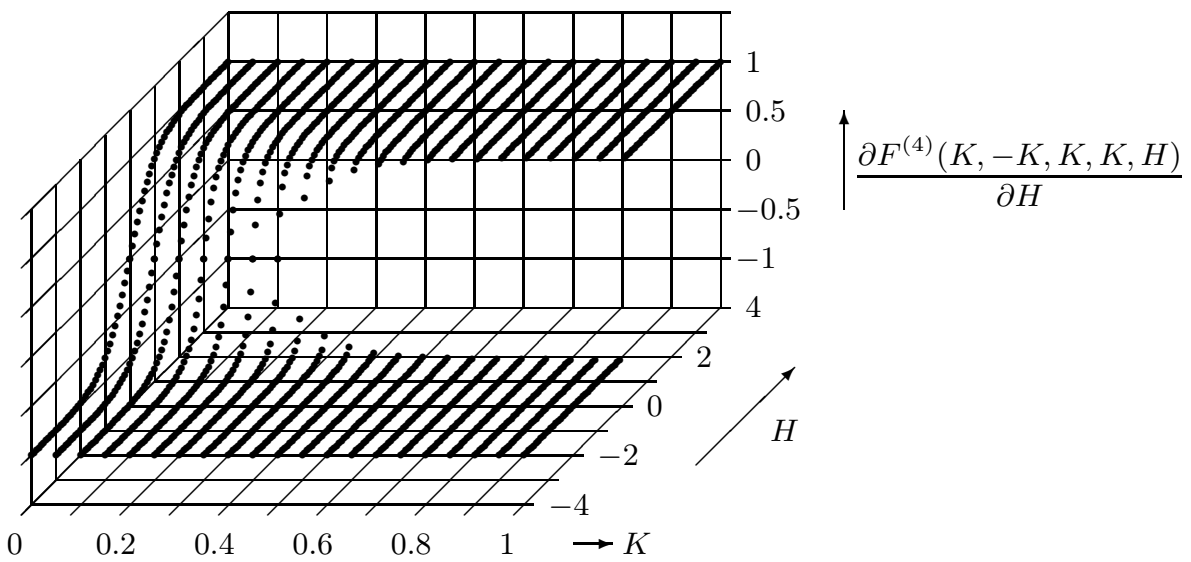

Рис. 5. Намагниченность магнетиков типа FA $\| \mathbf{F F}$.

Мы продемонстрировали глобальное поведение намагниченности всех девяти типов магнетиков, при этом обнаружили ее разрывы первого рода в критической точке для магнетиков типов $\mathbf{F F}\|\mathbf{F F}, \mathbf{F A}\| \mathbf{F F}$ при $H=0$, а для магнетиков типов $\mathbf{F F} \| \mathbf{F A}$, $\mathbf{A A}|| \mathbf{F F}$ и с несколько меньшей долей уверенности для $\mathbf{F F} \| \mathbf{A A}-$ при $H \neq 0$. Особенности для других типов имеют меньший порядок. Чрезвычайно трудно было обнаружить особенность у магнетика типа $\mathbf{A A}|| \mathbf{A A}$, для которого по нашим данным лишь пятая производная $\partial^{5} F^{(4)}(-K,-K,-K,-K, H) / \partial H^{5}$ претерпевает разрыв.

Важным является также то, что при перестановке двух групп параметров происходит не только количественное изменение поведения их макрохарактеристик, но может происходить, как это не покажется на первый взгляд странным, качественное изменение поведения (см., например, рис. 5, 6 с качественно различными графиками 


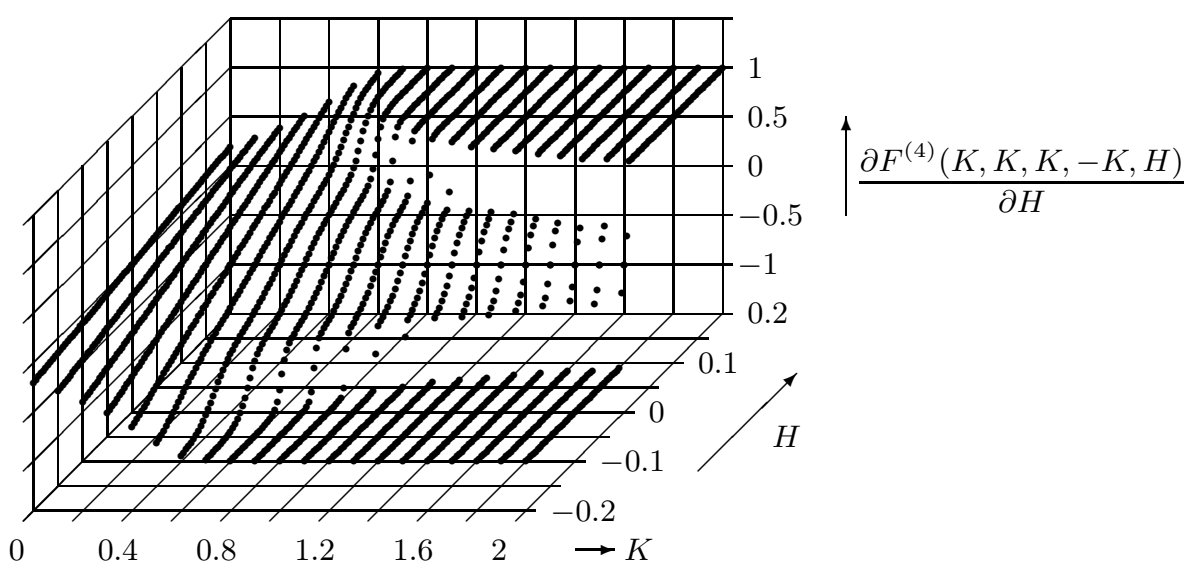

Рис. 6. Намагниченность магнетиков типа FF $\mid$ FA.

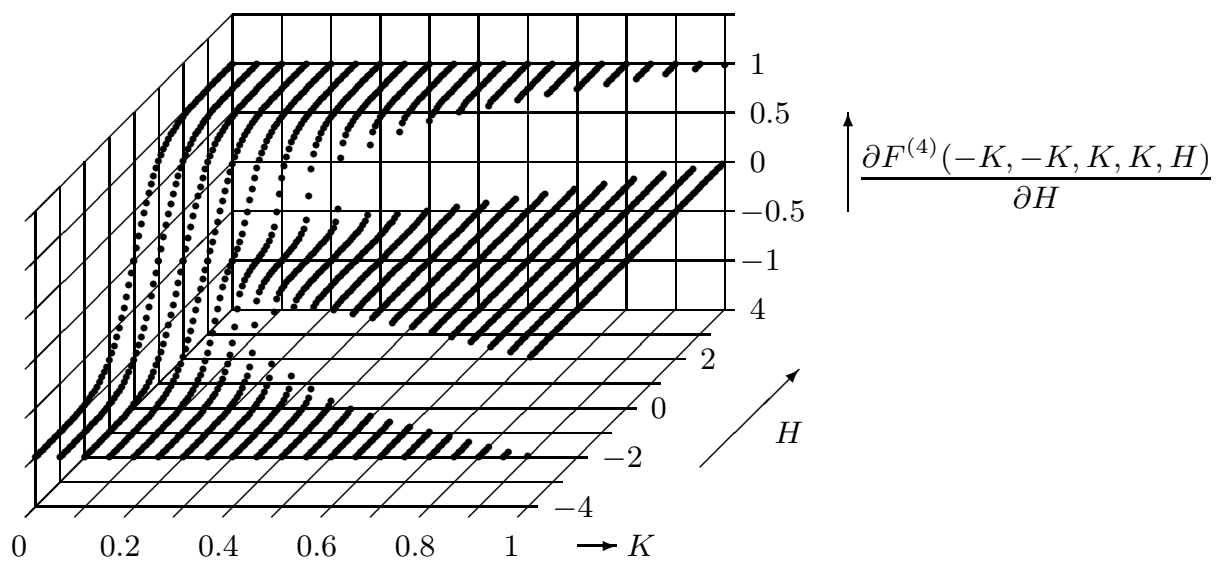

Рис. 7. Намагниченность магнетиков типа AA $\| \mathbf{F F}$.

намагниченности $\mathrm{FA}|| \mathrm{FF}$ - и FF || $\mathrm{FA}$-магнетиков). Это еще раз говорит об отсутствии симметрии между двумя группами параметров.

\section{4. СИНГУЛЯРНЫЕ ГИПЕРБОЛЫ МАГНЕТИКОВ}

Под сингулярными точками свободной энергии мы понимаем точки $(K ; H)$, в которых она не (вещественно) аналитична. Проведенные здесь и ранее исследования [5] показали существование для каждой линейности и каждого типа магнетиков соответствующего множества сингулярных точек, образующих некоторую кривую, которую будем называть сингулярной вслед за автором книги [7]. Так как сингулярная кривая всегда имеет прямолинейную асимптоту, к которой сингулярная кривая приближается монотонно (в некоторых случаях кривая совпадает с асимптотой), то сингулярную кривую мы будем также называть сингулярной гиперболой. Крайнюю 

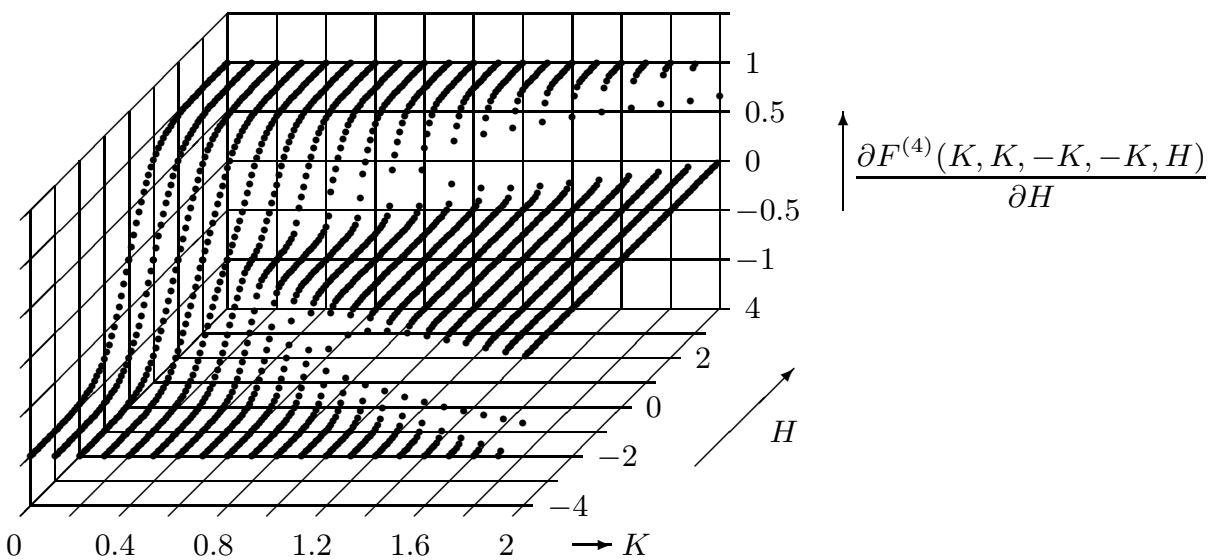

Рис. 8. Намагниченность магнетиков типа $\mathbf{F F} \| \mathbf{A A}$.

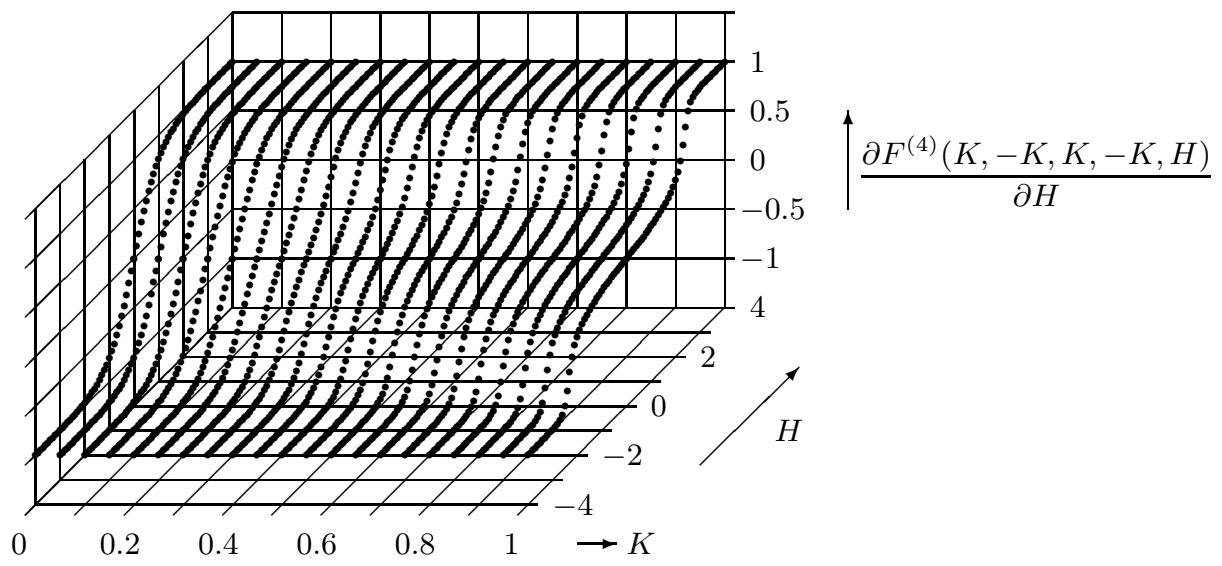

Рис. 9. Намагниченность магнетиков типа FA $\mid$ FA.

левую точку (точку с наименьшим значением координаты $K$ ), или, условно говоря, начальную точку сингулярной гиперболы, мы называем критической и обозначаем через $P_{\text {crit }}=\left(K_{\text {crit }} ; H_{\text {crit }}\right)$.

Далее мы приводим общее положение сингулярных гипербол, ограничиваясь при этом случаем $H \geqslant 0$, поскольку на отрицательные значения $H$ эти гиперболы продолжаются зеркальным отражением относительно горизонтальной оси.

Все числовые данные для построения сингулярных гипербол двух- и трехлинейных магнетиков взяты из работ [5], а для четырехлинейных магнетиков - из вышеприведенных данных, уточненных с привлечением данных (которые мы опустим из-за ограниченности объема статьи) о поведении производных высших порядков магнитной восприимчивости, псевдотеплоемкости и т.д. 


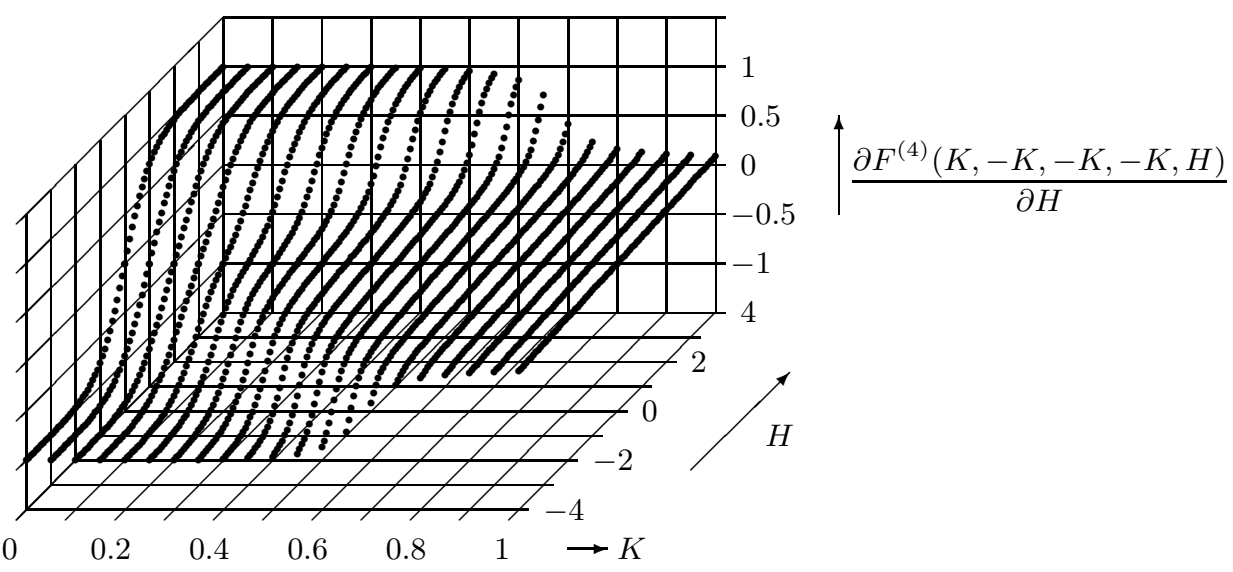

Рис. 10. Намагниченность магнетиков типа $\mathbf{F A} \| \mathbf{A A}$.

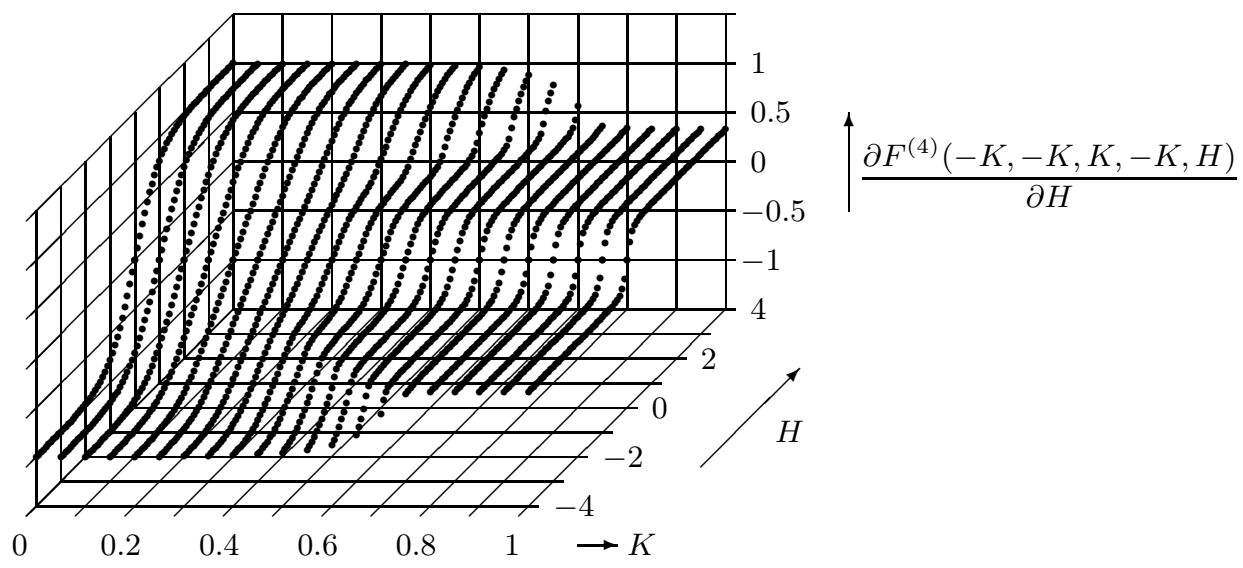

Рис. 11. Намагниченность магнетиков типа AA $\| \mathbf{F A . ~}$

4.1. Двухлинейные магнетики. Сингулярные гиперболы двухлинейных магнетиков имеют вид, представленный на рис. 13.

Видно, что у двухлинейных магнетиков всех типов есть всего одна критическая точка, и она расположена на оси $H=0$. При этом у магнетика типа $\mathbf{F F}$ на сингулярной гиперболе (в данном случае, на луче) намагниченность претерпевает разрыв первого рода в направлении $H$, а в критической точке псевдотеплоемкость обращается в бесконечность.

Для двух других типов магнетиков наши исследования показали, что псевдотеплоемкость обращается в бесконечность на обеих соответствующих гиперболах.

4.2. Трехлинейные магнетики. Картина расположения сингулярных гипербол у трехлинейных магнетиков более разнообразна, что иллюстрирует рис. 14 . 


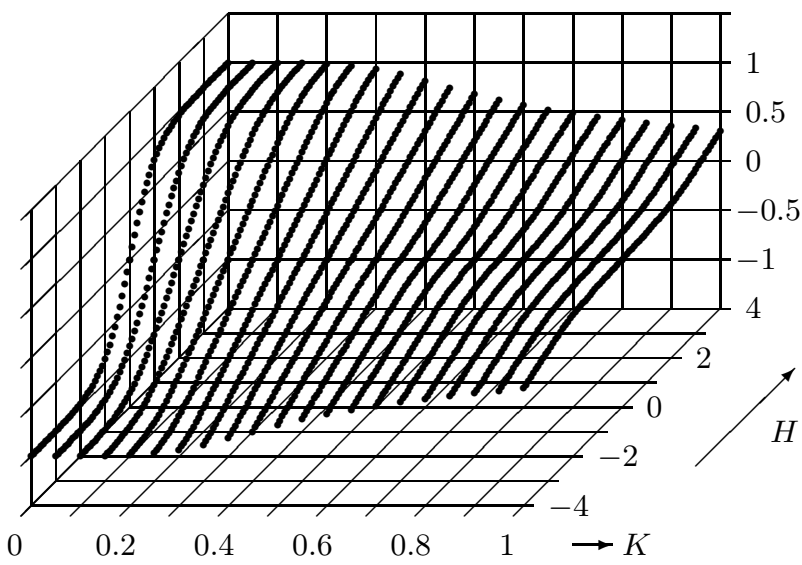

$$
\uparrow \frac{\partial F^{(4)}(-K,-K,-K,-K, H)}{\partial H}
$$

Рис. 12. Намагниченность магнетиков типа $\mathbf{A A} \| \mathbf{A A}$.

У магнетика типа $\mathbf{F F F}$, как и у двухлинейного магнетика $\mathbf{F F}$, на сингулярной кривой (луче) намагниченность претерпевает разрыв первого рода в направлении $H$, а в критической точке псевдотеплоемкость обращается в бесконечность.

У магнетика типа FAA критическая точка совпадает с критической точкой магнетика типа $\mathbf{F F F}$, а на сингулярной гиперболе псевдотеплоемкость обращается в бесконечность.

У двух других типов магнетиков на прямой $H=0$ нет сингулярных точек.

У магнетика типа АAA критическая точка расположена в области $H>0$, а на сингулярной гиперболе псевдотеплоемкость обращается в бесконечность.

У магнетика типа FFA критическая точка расположена в области $H>0$, а на сингулярной гиперболе в бесконечность обращается магнитная восприимчивость.

4.3. Четырехлинейные магнетики. Еще более сложную структуру расположения сингулярных гиперболах имеют четырехлинейные магнетики (рис. 15).

Поясним, что на этом рисунке все сингулярные гиперболы промаркированы в их конце за исключением двух, которые промаркированы в их начале. Светлые кружки отвечают магнетику типа $\mathbf{F F}|| \mathbf{F A}$, при этом для наглядности ординаты точек в этом случае увеличены в 10 раз, так что настоящая ордината начальной (критической) точки $\approx 0.1$.

Большими кружками, как и на двух предыдущих рисунках, отмечены начальные (критические) точки сингулярных гипербол.

Для трех типов магнетиков, свободная энергия которых не имеет сингулярностей при нулевом значении полевого параметра, критические точки расположены в области с ненулевым значением этого параметра. 


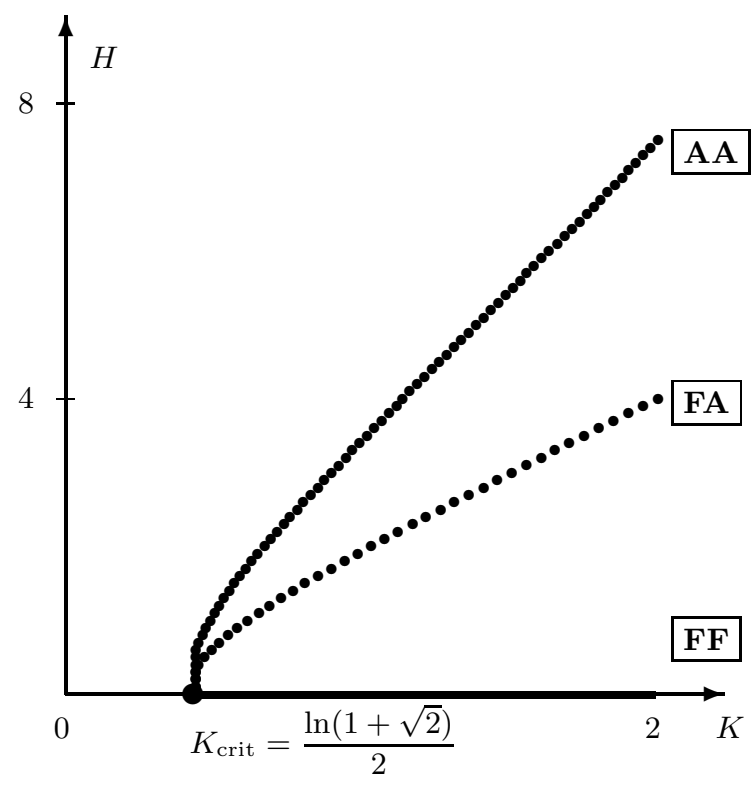

Рис. 13. Сингулярные гиперболы для трех типов двухлинейных магнетиков.

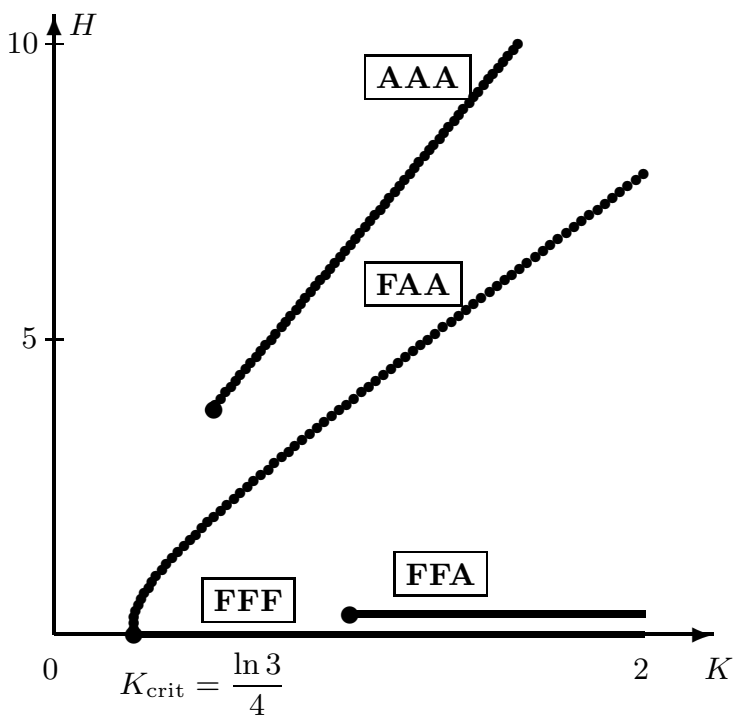

Рис. 14. Сингулярные гиперболы для четырех типов трехлинейных магнетиков.

\section{5. ЗАКЛЮЧЕНИЕ}

Все упомянутые выше теоретические результаты входят в созданную авторами теорию КТМ. Численные алгоритмы, разработанные на базе этой теории, оказались достаточно эффективными и позволили обнаружить весьма тонкие новые эффекты 


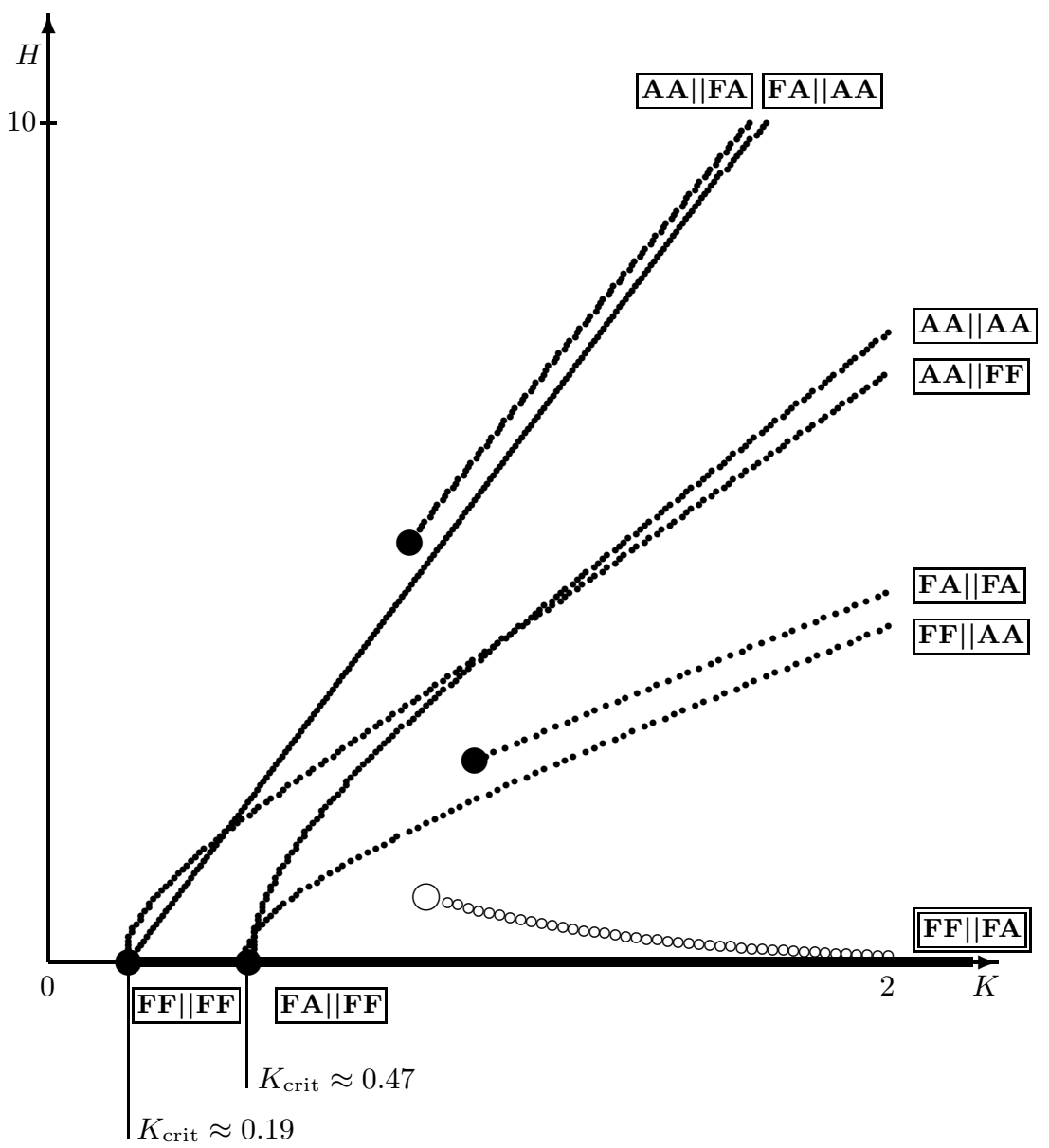

Рис. 15. Сингулярные гиперболы для девяти типов магнетиков.

в поведении макрохарактеристик изучаемых моделей. К ним нужно отнести полное описание глобального поведения макрохарактеристик, обнаружение для всех типов магнетиков сингулярных гипербол, их расположение и положение критических точек на них.

Вместе с тем к полученным численным результатам нужно относиться с известной осторожностью. Так, общее расположение сингулярных гипербол нами определено достаточно точно, что нельзя сказать о критических точках тех типов магнетиков, у которых она лежит в области, где $H \neq 0$, для их более точной локализации необходимо провести дополнительные исследования. Кроме того, не всегда точно можно определить порядок особенностей в сингулярных точках.

Тем не менее мы считаем, что качественное поведение основных макрохарактеристик магнетиков выявлено нами точно. Учитывая абстрактность моделей Изинга, 
которые лишь приближенно описывают реальные процессы, мы надеемся, что полученные нами численные результаты представляют интерес для приложений.

\section{Список литературы}

[1] А.А. Белавин, Лекиии по теории точно решаемых моделей квантовой теории поля, ч. І, МЦНМО, М., 1998; А. А. Белавин, А. Г. Кулаков, Р. А. Усманов, Лекции по теоретической физике, МЦНМО, М., 2001; Р. Бэкстер, Точно решаемые модели в статистической механике, Мир, М., 1985; М. Джимбо, Т. Мива, Алгебраический анализ точно решаемых моделей, Удмурдск. ун-т, Ижевск, 2000.

[2] А. А. Дмитриев, В. В. Катрахов, Ю.Н. Харченко, Корневые трансфер-матричъи в моделях Изинга, Наука, М., 2004.

[3] М. Фишер, Природа критического состояния, Мир, М., 1968.

[4] В. В. Катрахов, Ю. Н. Харченко, К. Ю. Тихонов, Корневые трансфер-матрицы многолинейных двумерных моделей типа Изинга. II. Статистическая сумма и КТМ четырехлинейных моделей, Препринт № 9 Ин-та прикл. матем. ДВО РАН, Дальнаука, Владивосток, 2005.

[5] В.В. Катрахов, Ю.Н. Харченко, К.Ю. Тихонов, Исследование многолинейных двумерных моделей типа Изинга методом корневых трансфер-матрии. І. Двухлинейные модели, Препринт № 3 Ин-та прикл. матем. ДВО РАН, Дальнаука, Владивосток, 2005; Исследование многолинейных двумерных моделей типа Изинга методом корневых трансфер-матрии. І. Трехлинейные модели, Препринт № 4 Ин-та прикл. матем. ДВО РАН, Дальнаука, Владивосток, 2005.

[6] В. В. Катрахов, Ю.Н. Харченко, Матем. моделир., 17:11 (2005), 72.

[7] Р.А. Минлос, Введение в математическую статистическую физику, МЦНМО, М., 2002. 